Bio - grafía. Escritos sobre la Biología y su Enseñanza. ISSN 2027-1034

Edición Extraordinaria. p.p. 984 - 991

Memorias del IX Encuentro Nacional de Experiencias en Enseñanza de la Biología y la Educación Ambiental. IV Congreso Nacional de Investigación en Enseñanza de la Biología.

\title{
CONSTRUCCIÓN DE UN RECURSO EDUCATIVO PARA LA ENSEÑANZA DE MICROALGAS ORIENTADO A ESTUDIANTES DE GRADO CUARTO DEL INSTITUTO PEDAGOGICO NACIONAL
}

Paula Milena Parra Rivera ${ }^{1}$

\section{RESUMEN}

El presente trabajo de grado, tuvo como objetivo construir un recurso educativo para la enseñanza de microalgas orientado a estudiantes de grado cuarto del Instituto Pedagógico Nacional situado en la ciudad de Bogotá, para ello se realizó la siembra de dos taxones de microalgas: Chlorella sp. y Scenedesmus sp, proporcionadas por la Línea de Enseñanza Aprendizaje de la Botánica de la Universidad Pedagógica

Nacional. Atendiendo a lo anterior, este trabajo se desarrolló bajo el enfoque de investigación mixto, lo cual logró un análisis con mayor profundidad a partir de los instrumentos utilizados, posibilitando el contraste frente a las ideas previas y lo que aprendieron los estudiantes alrededor de las microalgas y sus funciones vitales, a partir de lo anterior se diseña una cartilla denominada "conociendo el mundo microscópico", donde se abordaron diferentes temáticas, desde el buen uso del microscopio hasta las funciones vitales desarrolladas por estos organismos. Dentro del contexto educativo y de la misma enseñanza de la biología, se posibilitó el aprendizaje y la comprensión de varios conocimientos de la vida unicelular en los estudiantes de grado cuarto y de igual manera se potencializó actitudes procedimentales frente al uso adecuado del microscopio y aspectos actitudinales como la participación y escucha activa en clase.

PALABRAS CLAVE: Recurso educativo, enseñanza, microalgas, funciones vitales.

\section{ABSTRACT:}

The present work of degree, had the objective of constructing an educational resource for the teaching of microalgae directed to fourth grade students of the National Pedagogical Institute located in the city of Bogota, for it was done the sowing of two microalgae taxa: Chlorella sp. and Scenedesmus sp, provided by the Teaching Learning Line of Botany of the Pedagogical University

National. In view of the above, this work was developed under the mixed research approach, which achieved a deeper analysis based on the instruments used, allowing the contrast with previous ideas and learned students about the microalgae their functions

\footnotetext{
${ }^{1}$ Licenciada en Biología Universidad Pedagógica Nacional. Docente biología y Ed. Ambiental Instituto Pedagógico Nacional. Correo de contacto: pmparrar@pedagogica.edu.co.
} 
Bio - grafía. Escritos sobre la Biología y su Enseñanza. ISSN 2027-1034

Edición Extraordinaria. p.p. 984 - 991

Memorias del IX Encuentro Nacional de Experiencias en Enseñanza de la Biología y la Educación Ambiental. IV Congreso Nacional de Investigación en Enseñanza de la Biología.

vital, from the previous use of the microscope to the vital functions developed by these organisms. Within the educational context and of the same teaching of biology, it allowed the learning and the understanding of several knowledge of the unicellular life in the students of fourth degree and of equal way it was put forward procedural attitudes against the acceptable use of the microscope and attitudinal ones like participation and active listening

in

class.

\section{KEYWORDS}

Educational resource, teaching, microalgae, vital functions.

\section{INTRODUCCIÓN}

La Universidad Pedagógica Nacional, desde su misión propone la formación de profesionales de la educación capaces de investigar y difundir conocimientos pedagógicos y didácticos que consoliden una interacción directa con la sociedad, esto quiere decir que la investigación forma parte fundamental en los maestros ya que logran identificar y reconocer fortalezas y debilidades según la disciplina en la que se está formando y así contribuir a una sociedad mejor. Dado lo anterior, se cumple uno de los objetivos que propone la Universidad Pedagógica Nacional y es el de producir conocimientos en el ámbito educativo y pedagógico que involucre procesos de innovación educativa, esto se logra a partir de la estructuración del plan curricular y el enfoque de las líneas de investigación en este caso de la licenciatura en Biología, el cual implica para los estudiantes realizar prácticas pedagógicas, lo cual permite en los maestros en formación ampliar la visión que se tiene en el contexto educativo, evidenciando problemáticas o fortalezas que posee la enseñanza de la biología dentro de diferentes instituciones educativas del país.

De acuerdo a lo anterior, se puede establecer características que posee el enseñar biología, uno de ellos se establece desde la ampliación del conocimiento a partir del estudio de las comunidades biológicas privilegiando el campo disciplinar y otros desde el campo pedagógico y didáctico proponiendo estrategias de enseñanza y aprendizaje, todo esto va retroalimentando y consolidando varias posiciones dentro del cómo se debe enseñar la biología, pero no se puede descubrir esto si antes no se ha logrado una interacción en el campo educativo teniendo en cuenta los procesos cognitivos de los estudiantes y de su actuar en el contexto que lo rodea, por lo anterior desde el PCLB se propone un año de prácticas pedagógicas y es desde allí que se empieza a estructurar este trabajo de grado.

Durante la práctica pedagógica que se desarrolló en el Instituto Pedagógico Nacional con los estudiantes de cuarto de primaria, se evidenció la dificultad de comprender la vida microscópica entorno a las microalgas que forman parte primordial en los ecosistemas acuáticos, de acuerdo a estas dificultades se diseña una cartilla con varias actividades, lo 
Bio - grafía. Escritos sobre la Biología y su Enseñanza. ISSN 2027-1034

Edición Extraordinaria. p.p. 984 - 991

Memorias del IX Encuentro Nacional de Experiencias en Enseñanza de la Biología y la Educación Ambiental. IV Congreso Nacional de Investigación en Enseñanza de la Biología.

cual proporcionó en los estudiantes un acercamiento mayor a estos organismos, resaltando la importancia de que estos son organismos clorofilicos.

Esta cartilla da cuenta del enfoque pedagógico establecido dentro del Instituto Pedagógico Nacional denominado "inteligencias múltiples" donde el estudiante tiene la posibilidad de interpretar el mundo desde diferentes maneras, es allí donde el recurso educativo es importante ya que el niño tiene la posibilidad de interactuar, discutir y reflexionar sobre la importancia de estos seres vivos en los ecosistemas acuáticos.

\section{METODOLOGÍA}

A continuación, se presenta un esquema donde se evidencia a manera global, el paso a paso de esta investigación:

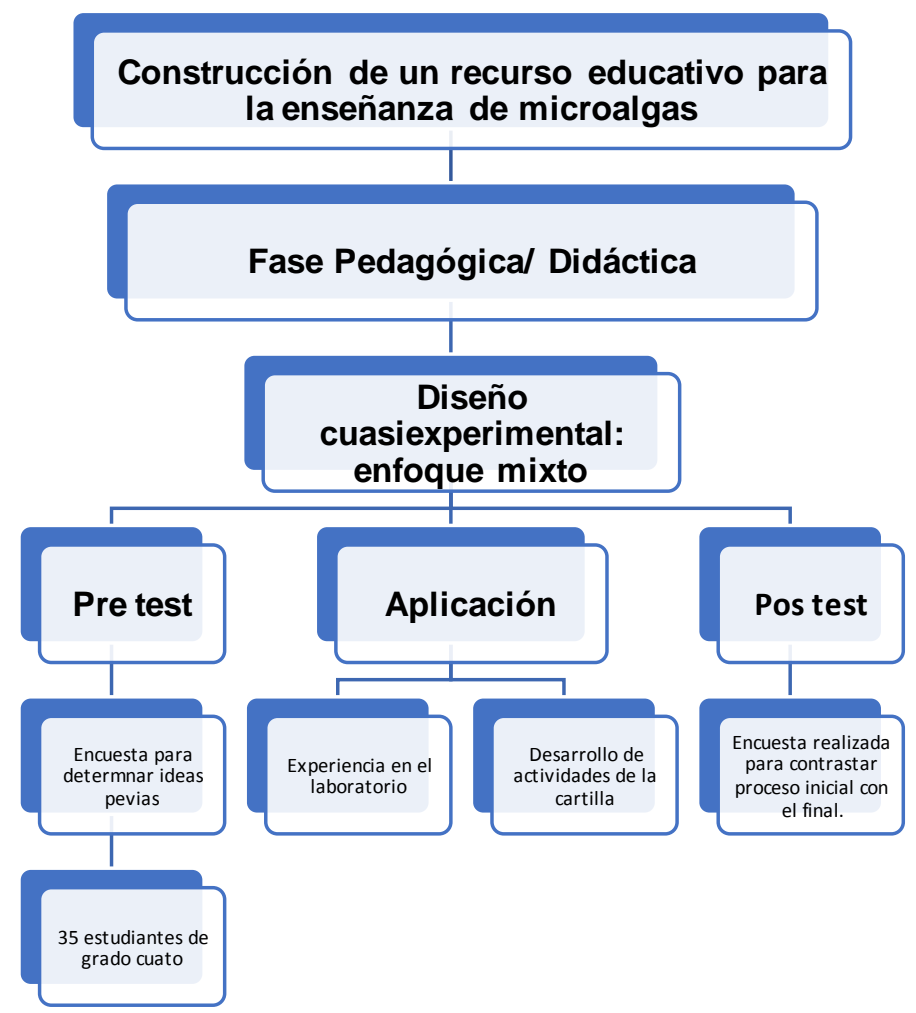

Figura 1. Metodología de investigación. Elaboración propia.

De acuerdo a lo anterior, este trabajo se desarrolló bajo el enfoque de investigación mixto, el cual tiene en cuenta un proceso de recolecta, análisis y vinculación de datos cuantitativos y cualitativos para un mismo estudio, además de esto, permite tener formas apropiadas para estudiar y teorizar los problemas de investigación, esto produce datos 
Bio - grafía. Escritos sobre la Biología y su Enseñanza. ISSN 2027-1034

\section{Edición Extraordinaria. p.p. 984 - 991}

Memorias del IX Encuentro Nacional de Experiencias en Enseñanza de la Biología y la Educación Ambiental. IV Congreso Nacional de Investigación en Enseñanza de la Biología.

más completos, ya que se consideran diversas fuentes, contextos y análisis (Gómez, 2010. Pág. 72). De acuerdo a lo anterior, este tipo de enfoque representa procesos sistemáticos, empíricos y críticos que implican la recolección y el análisis de datos desde la mirada cuantitativa y cualitativa, así como su integración y discusión conjunta, lo que aporta fluidez al momento de sistematizar lo realizado entorno a las microalgas y su viabilidad en la enseñanza de la biología.

Para la construcción de la cartilla, se tuvo en cuenta el modelo constructivista tomando como parte importante la relación del sujeto con su contexto, concibiendo al estudiante como una persona capaz de tener reflexiones acerca de lo que vive y aprende, esto sin dejar a un lado el papel del maestro como aquel que orienta los conocimientos, esto fue fundamental al momento del diseño de la cartilla, ya que se estableció a partir de módulos educativos. También en este tipo de investigación

se trabajó el diseño cuasiexperimental, ya que se utilizó un pre test para conocer las ideas previas de los treinta y cinco estudiantes referente a las microalgas y el ecosistema donde habitan, después se realiza un pos-test, que tiene como finalidad reconocer una posible evolución conceptual en los estudiantes a través de los diferentes trabajos prácticos y experimentales ejecutados, ya que como menciona Campbell y Stanley (1996), estas prácticas permiten la evolución de los conocimientos adquiridos y al someterse estos mismos por segunda vez, suelen desempeñarse mejor a diferencia de la primera prueba. Para que este resultado sea visible, se recomienda que el estudiante tenga cierta privacidad en sus respuestas con los demás al momento de resolver las preguntas estructuradas.

Es importante mencionar que dentro de la investigación, se tuvo una constante actualización de la información por medio de bases de datos disponibles en la página web de la Universidad Pedagógica Nacional como EBSCO y google académico y la consulta de diferentes libros con las temáticas a tratar los cuales se enmarcan dentro de la ecología de los ecosistemas y generalidades de las microalgas y en la parte pedagógica se hizo la lectura de las fases que propone Piaget, modelo constructivista y lo que se entiende por un recurso educativo y de igual manera se hace la lectura de lineamientos y estándares educativos.

\section{RESULTADOS}

CATEGORIA

Superior

\section{OBSERVACIONES}

Presenta claridad en sus respuestas haciendo caso a la instrucción dada. Presenta mayor profundidad en sus definiciones al contemplar términos biológicos. 
Bio - grafía. Escritos sobre la Biología y su Enseñanza. ISSN 2027-1034

\section{Edición Extraordinaria. p.p. 984 - 991}

Memorias del IX Encuentro Nacional de Experiencias en Enseñanza de la Biología y la Educación Ambiental. IV Congreso Nacional de Investigación en Enseñanza de la Biología.

\begin{tabular}{|l|l|}
\hline Bueno & $\begin{array}{l}\text { Responde algunas preguntas con precisión } \\
\text { presentando coherencia en las respuestas dadas. }\end{array}$ \\
\hline Aceptable & $\begin{array}{l}\text { Responde de manera incompleta las preguntas } \\
\text { planteadas lo cual demuestra poco manejo de } \\
\text { términos biológicos. }\end{array}$ \\
\hline Deficiente & $\begin{array}{l}\text { No responde adecuadamente lo que se le plantea } \\
\text { en la guía de trabajo. }\end{array}$ \\
\hline
\end{tabular}

Figura 2. Tabla de categorías. Elaboración propia.

Según Campbell y Stanley (1966), es importante tener en cuenta el diseño antes y después por lo que se recomienda hacer el mismo para corroborar resultados. De acuerdo a esto, el pre-test y el post test se diseñó teniendo en cuenta las siguientes temáticas para determinar las ideas previas de los estudiantes, se plantean cinco preguntas donde los estudiantes tenían la posibilidad de escribir, dibujar e interpretar, lo cual se categorizó de la siguiente manera utilizando la siguiente tabla de desempeños:

De acuerdo a esta sistematización, se determina que 23/35 estudiantes se encuentran en nivel S (Superior), ya que distinguen en la naturaleza elementos vivos e inertes predominando en este segundo concepto elementos como ríos, piedras mientras que en organismos vivos prevalecen los animales que se pueden observar a simple vista como peces, mamíferos, aves, etc., Aunque los estudiantes identifican estos elementos, en sus respuestas se observa la tendencia a dibujar organismos grandes y con o que se encuentran familiarizados como los perros, gatos, entre otros, esto se debe a que como menciona Rivera, L (2013) en cuanto a que los estudiantes explican la vida según el movimiento y lo que ven a simple vista. En cuanto a la segunda pregunta, donde el estudiante debía especificar las sustancias que necesitan los organismos clorofilicos para sobrevivir dentro del ecosistema, 30/35 estudiantes, se encuentran en el nivel B ya que no logan identificar claramente los compuestos que necesitan específicamente los organismos clorofílicos,

Al momento de preguntar a los estudiantes la definición de que era un organismo microscópico, 31/35 estudiantes se encontraron en el nivel $\mathbf{S}$ al mencionar que son seres vivos que no se pueden observar a simple vista por lo que se necesitan observar con un instrumento óptico como el microscopio. En cuanto al reconocimiento por medio de imágenes de organismos microscópicos, 22/35 estudiantes se encuentran en nivel S, ya que a partir de la actividad identificaron diversos representantes del reino monera. La quinta pregunta en la cual los estudiantes debían reconocer la importancia del intercambio de materia y energía para organismos en ecosistemas acuáticos, 19/35 estudiantes dibujaron peces, tortugas mencionando que se alimentaban de microorganismos y de algas que se pueden ver a simple vista. 
Bio - grafía. Escritos sobre la Biología y su Enseñanza. ISSN 2027-1034

Edición Extraordinaria. p.p. 984 - 991

Memorias del IX Encuentro Nacional de Experiencias en Enseñanza de la Biología y la

Educación Ambiental. IV Congreso Nacional de Investigación en Enseñanza de la Biología.

De acuerdo al puntaje de la última pregunta, se deduce que la palabra "microalga" parece ser sencilla, pero se muestra confusión ya que los estudiantes no logran establecer una relación directa con las algas desde una mirada microscópica, al momento de solicitar alguna definición acerca de estos organismos, ninguno daba una respuesta correcta, esto se debe a que no han tenido un acercamiento a estos organismos ni en la escuela ni en su vida cotidiana.

A partir de los análisis del pre- test, se diseñaron en total tres módulos educativos, donde se tuvo en cuenta la lectura del plan de área de Ciencias Naturales y Educación Ambiental, estándares y lineamientos curriculares, en la figura tres, se muestran los contenidos y el trabajo realizado por parte de los estudiantes:
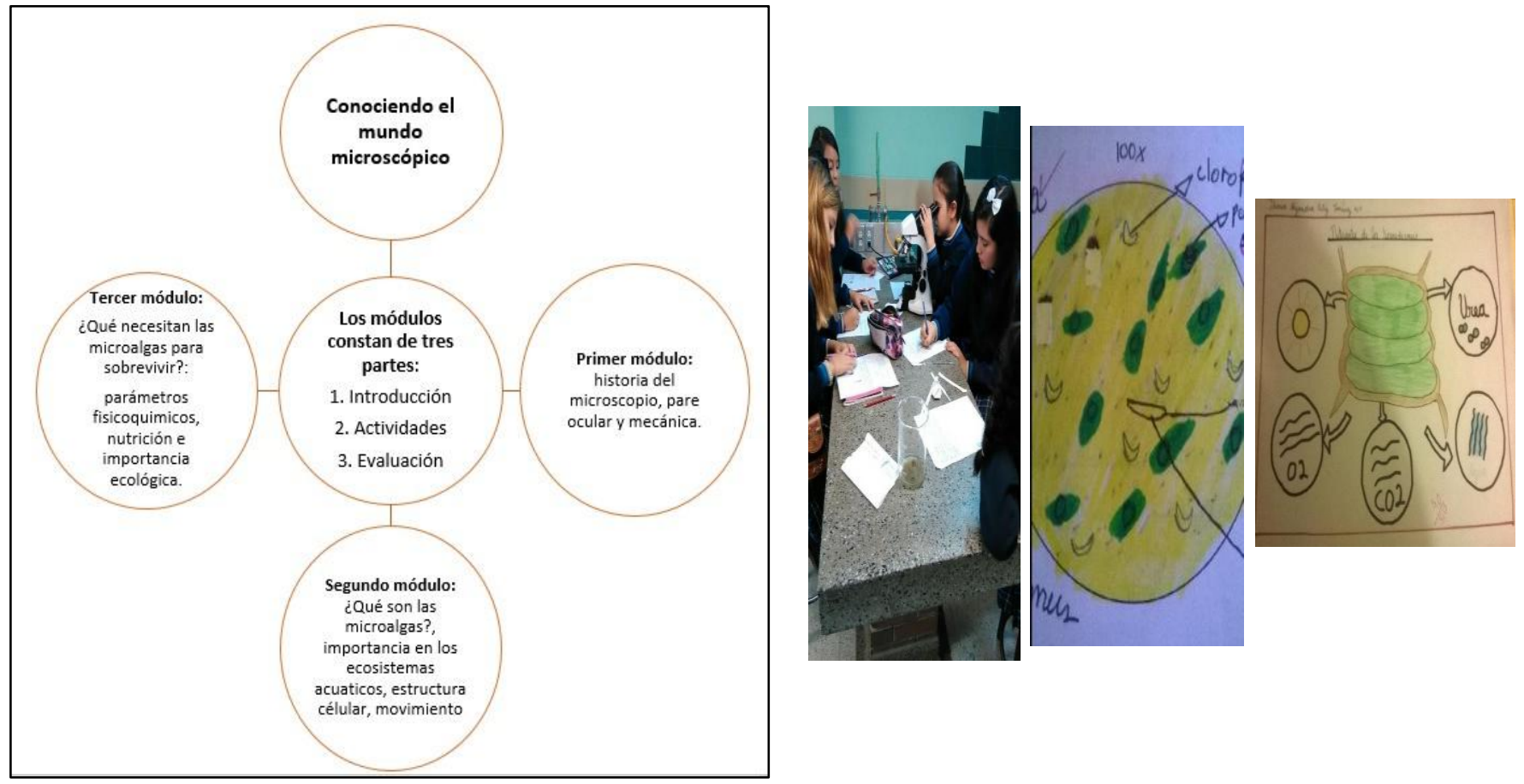

Figura 3. Contenidos de la cartilla educativa: "conociendo el mundo microscópico. Elaboración propia.

Al momento de realizar el pos test y de su calificación, se demuestra de manera cualitativa que los estudiantes destacan en cada trabajo, la distinción que hacen de las microalgas a partir de su estructura celular, reconocen que son del reino protista y que llevan a cabo el proceso de fotosíntesis, reconocen las características claves que poseen los organismos microscópicos y reconocen caracteres importantes en cuanto a la morfología de Chlorella sp. especificando forma esferoidal, su color y su cloroplasto en forma de copa gracias a la observación en 100X, en el caso de Scenedesmus sp., los estudiantes reconocieron la presencia de flagelos en los extremos de las colonias y su 
Bio - grafía. Escritos sobre la Biología y su Enseñanza. ISSN 2027-1034

\section{Edición Extraordinaria. p.p. 984 - 991}

Memorias del IX Encuentro Nacional de Experiencias en Enseñanza de la Biología y la Educación Ambiental. IV Congreso Nacional de Investigación en Enseñanza de la Biología.

forma alargada, además de ello distinguieron las colonias de estos organismos clorofílicos. Es importante recalcar que, a partir de este trabajo, los estudiantes de grado cuarto tuvieron un primer acercamiento a la temática de microscopia, aprendieron las partes del microscopio tanto ópticas como mecánicas, resaltando la importancia de la observación generando comparaciones entre color, forma y tamaño de las microalgas, esto lo corrobora también Durango, M (2010), al decir que a partir de la microscopia, los estudiantes logran comprender de una manera significativa el concepto de célula además de la importancia de aplicar actividades didácticas permitiendo que el estudiante interactúe, participe y se intrigue aún más por el objeto de estudio.

\section{CONCLUSIONES}

1. La enseñanza de microalgas a estudiantes de cuarto de primaria es relevante en el entorno escolar, ya que desde esta edad es propicio que tengan un acercamiento hacia la vida microscópica, diferenciando formas y estructuras, además de ello se logró que los estudiantes manipularan de una manera responsable elementos de laboratorio como el microscopio electrónico y muestras algales.

2. Los estudiantes lograron reconocer que al igual que otros seres vivos como los mamíferos, reptiles 0 aves que las microalgas a partir de una sola célula pueden hacer diferentes funciones como nutrición o respiración, a partir de ello reconocieron la estructura celular de las microalgas logrando observar en el caso de Chlorella sp. su cloroplasto en forma de copa y en el caso de Scenedesmus sp. sus flagelos y forma alargada.

3. Es importante formar en los estudiantes de acuerdo a su etapa de desarrollo, la capacidad de observar muestras logrando diferenciar partes celulares específicas y dinámicas ecológicas de las microalgas en ecosistemas acuáticos.

4. Es importante que los maestros en Biología, logren diseñar diferentes recursos que potencialicen conocimiento en los estudiantes de acuerdo a las falencias que se observen en las dinámicas de las clases.

\section{BIBLIOGRAFIA}

Campbell y Stanley (1966). Diseños experimentales y cuasiexperimentales en la investigación social. Argentina.

Durango, M (2010). La microbiología en la escuela: una experiencia didáctica aplicada a séptimo grado de educación básica. Universidad Nacional de Colombia. Medellín: Colombia.

Gómez, L (2010). Construcción de escalas de evaluación e investigación. Departamento de Psicología, Universidad de Valladolid. Pág. 72 
Bio - grafía. Escritos sobre la Biología y su Enseñanza. ISSN 2027-1034

Edición Extraordinaria. p.p. 984 - 991

Memorias del IX Encuentro Nacional de Experiencias en Enseñanza de la Biología y la Educación Ambiental. IV Congreso Nacional de Investigación en Enseñanza de la Biología.

Ladino, N (2009). Paradigmas de investigación Enseñanza-Aprendizaje de la Botánica. universidad pedagógica nacional. Pág. 12.

Rivera, L (2013). Enseñanza aprendizaje del concepto de ser vivo en estudiantes de básica primaria. Universidad nacional de Colombia. Manizales, Colombia.

Universidad Pedagógica Nacional (2014). Plan de desarrollo institucional 2014-2019. Una universidad comprometida con la formación de maestros para una Colombia en paz. Tomado de: www.pedagogica.edu.co 